\title{
Do cholesterol and sphingomyelin change the mechanism of A $\beta 25-$ 35 peptide binding to zwitterionic bilayer?
}

\author{
Amy K. Smith, Elias Khayat, Christopher Lockhart, and Dmitri K. Klimov \\ School of Systems Biology, George Mason University, Manassas, VA 20110 \\ E-mail:dklimov@gmu.edu
}

\section{Supporting Information}

Selection of peptide force field: The selection of the all-atom CHARMM22 force field with CMAP corrections for amino acids was motivated by its ability to approximately reproduce the A $\beta 25-35$ secondary structure in the presence of SDS micelles [1].

Performance of REST algorithm: We used replica exchange with solute tempering (REST) molecular dynamics to sample the conformational ensemble of two A $\beta 25-35$ peptides binding to the ternary bilayer composed of DMPC, PSM, and cholesterol. REST utilized $R=6$ replicas, which were distributed in the temperature range from $330 \mathrm{~K}$ to $440 \mathrm{~K}$ (see Methods). To monitor replica mixing across the temperature range, we visualized the random walk of replicas over temperatures for each of six REST trajectories. As an example, the replica walk presented in Fig. S1 for one of the trajectories does not reveal systemic trapping of replicas at any temperature.

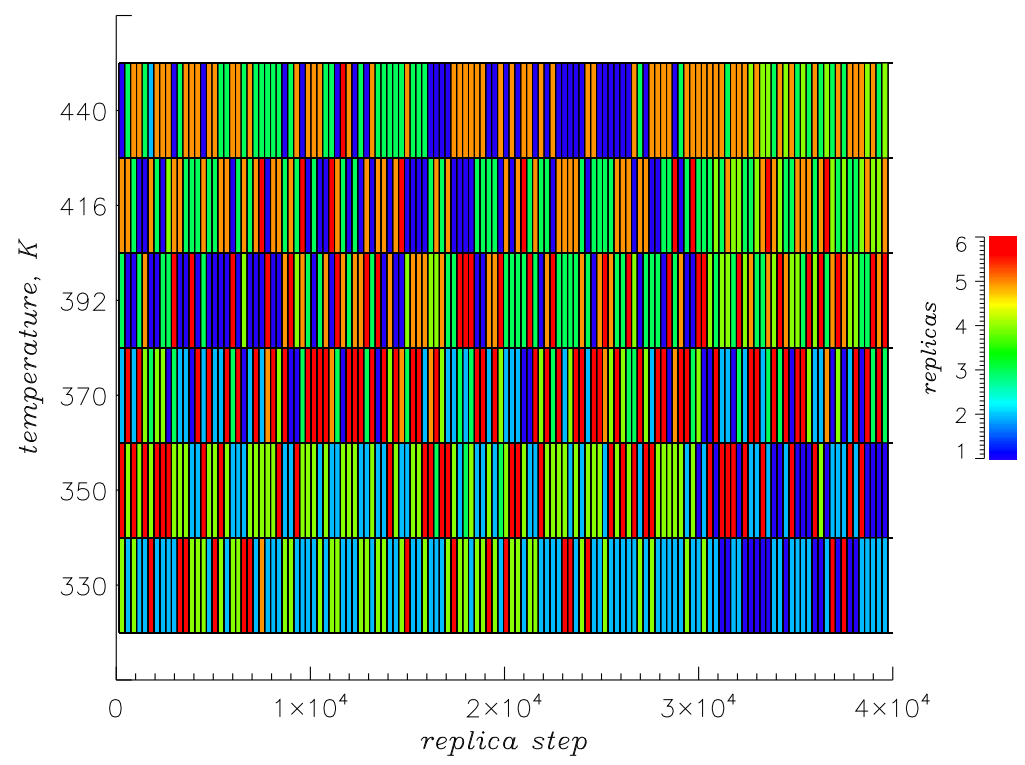

Figure S1. (a) Random replicas walk over temperatures in one of six REST trajectories. A color scale on the right codes the distribution of replicas over temperatures at the trajectory start. A color mosaic is indicative of efficient replica mixing.

A useful gauge of equal representation of all replicas at each temperature is a replica mixing parameter defined as [2]

$m(T)=1-\frac{\sqrt{\sum_{r=0}^{R-1} t_{r}^{2}}}{\sum_{r=0}^{R-1} t_{r}}$ 
where $T$ is the REST temperature and $t_{r}$ is the total number of REST iterations spent at $T$ by replica $r$. Assuming ideal mixing it is easy to derive an expression for optimum theoretical value of $m(T)=1-$ $1 / R^{I / 2}$, which is 0.59 for $R=6$. Fig. S2 shows that $m(T)$ is close to the theoretical value at most REST temperatures, whereas some deviations at the lowest and highest temperatures are due to boundary effects. Taken together, Figs. S1 and S2 suggest efficient replica mixing across temperatures, which is a necessary condition for REST simulation convergence.

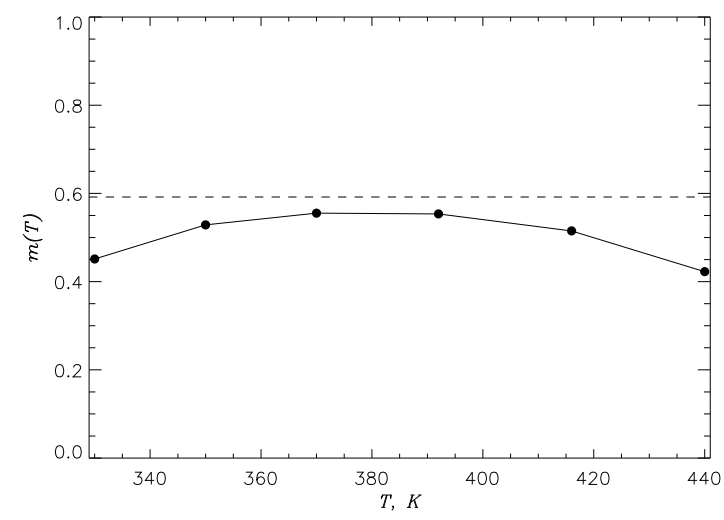

Figure S2. The replica mixing parameter $m(T)$ as a function of the REST temperature $T$. Dashed line indicates an optimum theoretical value of 0.59 . The data are averaged over six trajectories.

Convergence of REST conformational sampling: Each REST replica in each of six trajectories was sampled for $80 \mathrm{~ns}$ (see Methods). To evaluate equilibration of A $\beta 25-35$ peptide binding, we first considered the z-position of its center of mass $Z_{\text {com }}$ along the bilayer normal. The plot of $\left\langle Z_{\text {com }}(t)\right\rangle$ averaged over six trajectories as a function of REST sampling time $t$ at $330 \mathrm{~K}$ is presented in Fig. S3a. The figure suggests that $\left\langle Z_{\text {com }}(t)\right\rangle$ approximately reaches the equilibrium baseline after $t=\tau_{e q}=58 \mathrm{~ns}$. To test sampling convergence further, we computed the probability distributions $P\left(Z_{\text {com }}\right)$ using the data from all six REST trajectories and progressively eliminating the sampling from the beginning of simulations in the intervals of $2 \mathrm{~ns}$. Fig. S3b shows that the distributions $P\left(Z_{c o m}\right)$ become approximately stable after eliminating $\tau_{e q}=58 \mathrm{~ns}$ of initial sampling. Additionally, to map the convergence of bilayer structural quantities Fig. S3c,d present the bilayer area $\left\langle A_{b l}\right\rangle$ and bilayer thinning $\Delta D$. Both quantites show apparent convergence after $\tau_{e q}=58 \mathrm{~ns}$. 
(a)

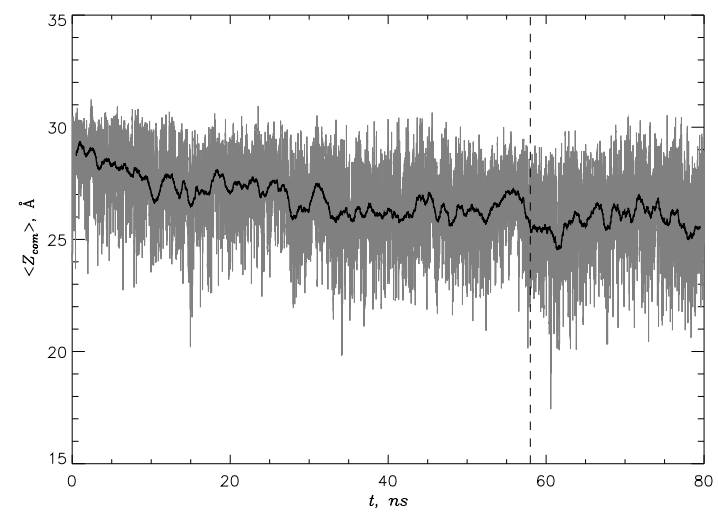

(c)

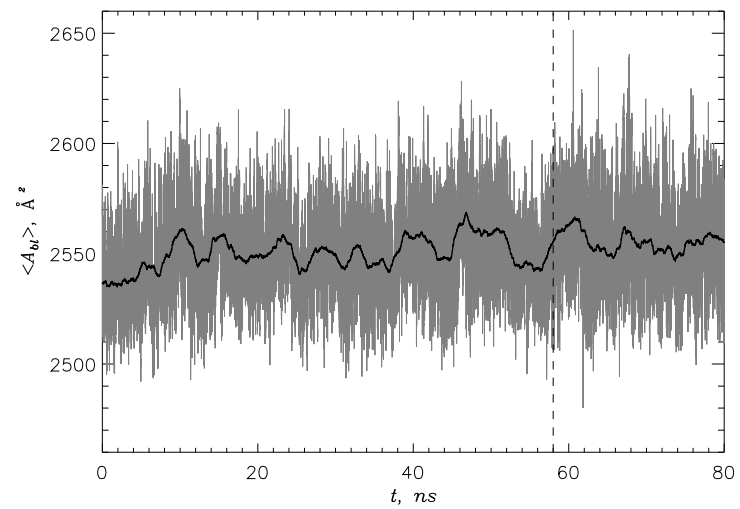

(b)

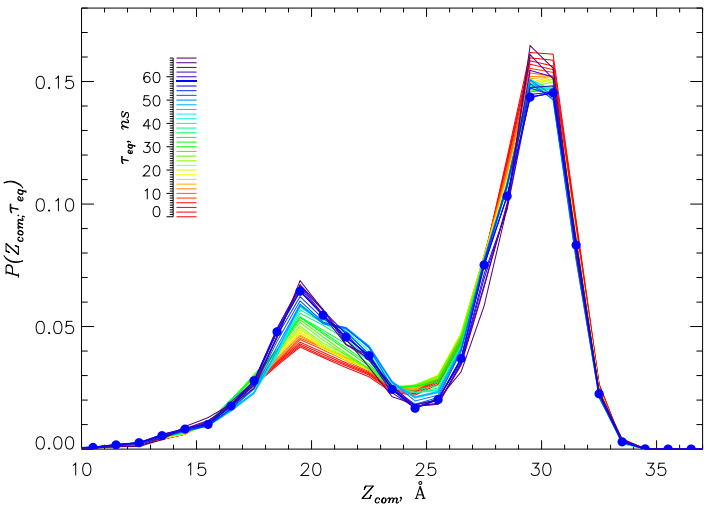

(d)

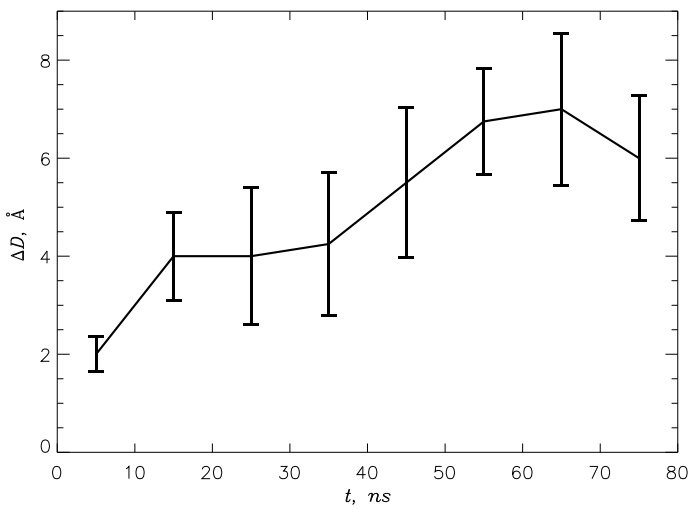

Figure S3. (a) The z-position of A $\beta 25-35$ peptide center of mass $\left\langle Z_{c o m}(t)\right\rangle$ along the bilayer normal is averaged over six trajectories and plotted as a function of REST sampling time $t$ at $330 \mathrm{~K}$. The equilibration time $\tau_{e q}=58 \mathrm{~ns}$ is marked by dashed line. Data in black and grey correspond to $\left\langle Z_{c o m}(t)\right\rangle$ processed with and without averaging sliding window of $1 \mathrm{~ns}$. (b) Probability distributions $P\left(Z_{c o m}\right)$ computed by excluding the initial sampling times at $330 \mathrm{~K}$ in the intervals of $2 \mathrm{~ns}$ up to $68 \mathrm{~ns}$. The first approximately stable distribution distinguished by thick blue line and solid circles corresponds to excluding $\tau_{e q}=58 \mathrm{~ns}$. The color scale codes the initial excluded time. (c-d) Additional measures of sampling convergence are provided by the bilayer area $\left\langle A_{b l}\right\rangle$ in (c) and bilayer thinning $\Delta D$ in (d) (see Methods for the definitions of $\Delta D$ ). $\left\langle A_{b l}\right\rangle$ is computed and plotted similar to $\left\langle Z_{c o m}(t)\right\rangle$ in (a), whereas $\triangle D$ is computed by block averaging the data over $10 \mathrm{~ns}$ and six trajectories. Panels (c-d) demonstrate that after $\tau_{e q}$ $=58 \mathrm{~ns}$ both quantities are approximately stable apart from some fluctuations.

To provide further tests of convergence of equilibrium REST sampling, we employed two approaches. First, following our previous studies [1] we enumerated unique conformational states $N_{s}$ collected as a function of the cumulative equilibrium simulation time $\tau_{s i m}$ in the wild-type replica at $330 \mathrm{~K}$. A unique state is determined by the enthalpy $H$ and a structural quantity $X$, which can be either the number of intrapeptide contacts $C$, the number of peptide-bilayer contacts $C_{l}$, or the distance between the peptide center of mass and the bilayer midplane $Z_{c o m}$. Enthalpy and $Z_{c o m}$ were binned using the intervals of 2 $\mathrm{kcal} / \mathrm{mol}$ and $1 \AA$, respectively. To eliminate the dependence of $N_{s}$ on the arbitrary order of state collection, we performed 200 random permutations of equilibrium structural snapshots to compute average $N_{s}\left(\tau_{s i m}\right)$. Fig. S4a demonstrates an approximate exhaustion of states in $N_{s}\left(\tau_{s i m}\right)$ collected using the three definitions. Such behavior is a prerequisite for sampling convergence. 
(a)

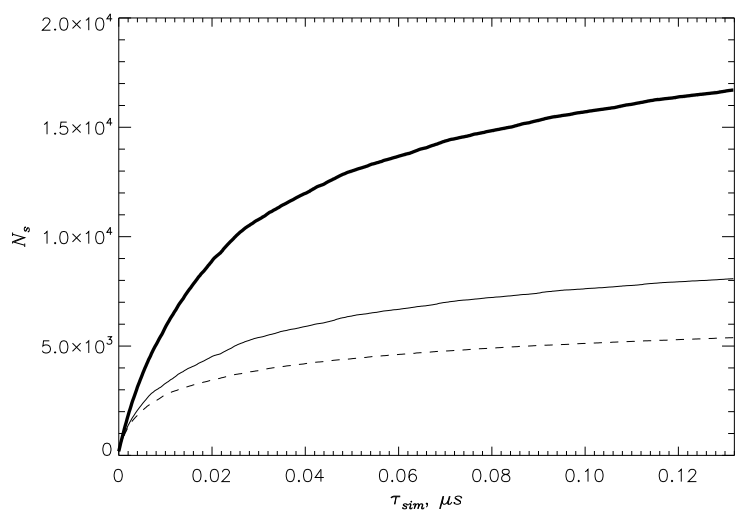

(b)

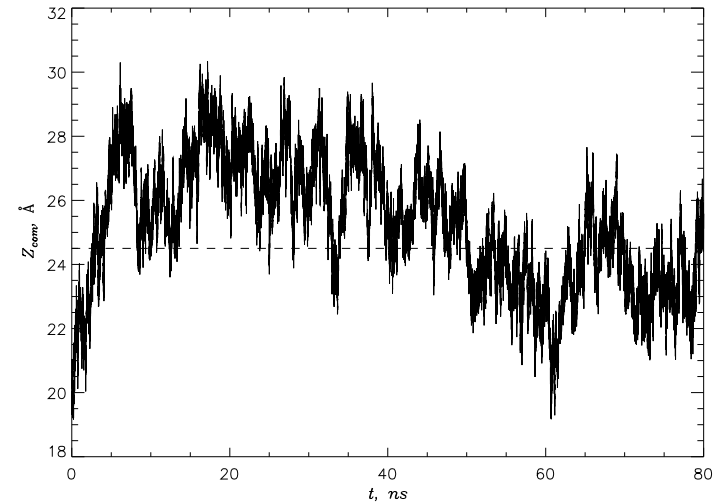

(c)

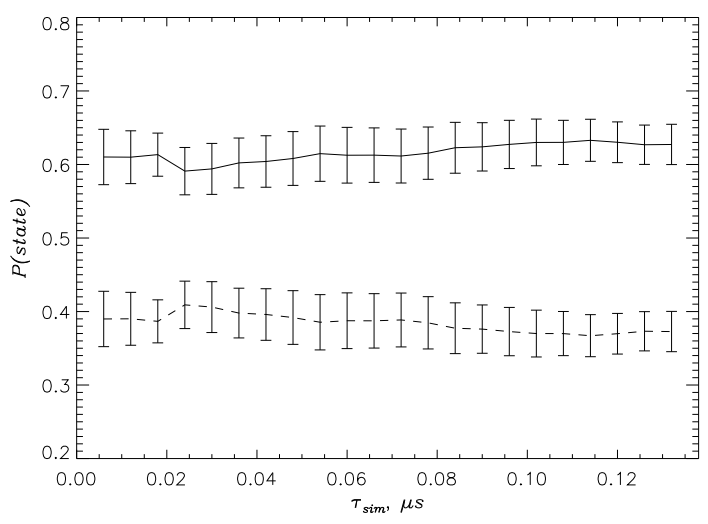

Figure S4. (a) The numbers of unique states $N_{s}$ collected at $330 \mathrm{~K}$ as a function of cumulative equilibrium simulation time $\tau_{\text {sim }}$. Thick, thin, and dashed lines mark $N_{s}$ defined using $\left(H, C_{l}\right),\left(H, Z_{c o m}\right)$, and $(H, C)$ states, respectively. (b) The z-position of A $\beta 25-35$ peptide center of mass $Z_{c o m}(t)$ in one of the REST replicas as a function of REST sampling time $t$. The value of $z$ separating inserted $\mathbf{I}$ and surface-bound $\mathbf{S}$ states (see Fig. 6) is shown by dashed line. (c) Probabilities of $\mathbf{I}$ and $\mathbf{S}$ states, $P(I)$ (dashed line) and $P(S)$ (solid line), as a function of cumulative equilibrium simulation time $\tau_{\text {sim }}$ at $330 \mathrm{~K}$. Sampling errors are given by vertical bars.

Second, in Results section of the main text we define two A $\beta 25-35$ binding states, inserted $\mathbf{I}$ and surfacebound $\mathbf{S}$. Fig. S $4 \mathrm{~b}$ presents the position along the z-axis of A $\beta 25-35$ peptide center of mass $Z_{c o m}(t)$ in one of the REST replicas as a function of REST sampling time $t$. The plot reveals transitions between $\mathbf{I}$ and $\mathbf{S}$ occurring during the walk of a replica over temperatures suggesting equilibration of the simulation system at different temperatures. To directly assess equilibrium sampling convergence, we considered the probabilities for A $\beta 25-35$ peptide to sample $\mathbf{I}$ and $\mathbf{S}, P(I)$ and $P(S)$, as a function of cumulative equilibrium simulation time $\tau_{\text {sim. }}$. In Fig. S4c after initial drift both $P(I)$ and $P(S)$ reach respective plateau values of $0.36 \pm 0.06$ and $0.64 \pm 0.06$ at about $100 \mathrm{~ns}$. It is important to note that the sampling errors are small enough to clearly distinguish $P(I)$ and $P(S)$. Taking multiple measures presented above together, we assume that the REST simulations of A $\beta 25-35$ binding to the ternary bilayer have converged.

Secondary structure in A $\beta 25-35$ peptide: The secondary structure propensities in A $\beta 25-35$ peptide and its regions bound to the ternary and DMPC bilayers are presented in Table S1. These data are discussed in the main text. 
Table S1 Secondary structure propensities in A $\beta 25-35$ peptide

\begin{tabular}{|c|c|c|c|c|}
\hline System & Quantity & Peptide & $\mathbf{R 3}$ & $\mathbf{R 4}$ \\
\hline \multirow{3}{*}{ ternary bilayer } & $\langle\boldsymbol{H}\rangle$ & $0.33 \pm 0.05$ & $0.25 \pm 0.06$ & $0.38 \pm 0.04$ \\
\cline { 2 - 5 } & $\langle\boldsymbol{T}\rangle$ & $0.36 \pm 0.04$ & $0.41 \pm 0.04$ & $0.32 \pm 0.04$ \\
\cline { 2 - 5 } & $\langle\boldsymbol{R C}\rangle$ & $0.31 \pm 0.01$ & $0.33 \pm 0.03$ & $0.30 \pm 0.02$ \\
\hline \multirow{3}{*}{ DMPC $^{a}$} & $\langle\boldsymbol{H}\rangle$ & $0.31 \pm 0.03$ & $0.18 \pm 0.03$ & $0.39 \pm 0.02$ \\
\cline { 2 - 5 } & $\langle\boldsymbol{T}\rangle$ & $0.36 \pm 0.02$ & $0.40 \pm 0.03$ & $0.34 \pm 0.02$ \\
\cline { 2 - 5 } & $\langle\boldsymbol{R C}\rangle$ & $0.33 \pm 0.02$ & $0.43 \pm 0.03$ & $0.28 \pm 0.02$ \\
\hline
\end{tabular}

adata from [1]

Tertiary interactions in A $\mathbf{\beta 2 5 - 3 5}$ peptide: To facilitate the analysis of tertiary interactions, we identified the most stable side chain contacts occurring in the A $325-35$ peptide bound to the ternary and pure DMPC bilayers (Tables S2 and S3). Their analysis and comparison are presented in the main text.

Table S2 Stable intrapeptide contacts in A $\beta 25-35$ monomer bound to the ternary bilayer

\begin{tabular}{|c|c|c|}
\hline Rank & Contact & $\langle\boldsymbol{C}(\boldsymbol{i}, \boldsymbol{j})\rangle$ \\
\hline 1 & Gly29-Ile32 & $0.68 \pm 0.07$ \\
\hline 2 & Gly29-Gly33 & $0.50 \pm 0.06$ \\
\hline 3 & Asp27-Ala30 & $0.47 \pm 0.07$ \\
\hline 4 & Ala30-Gly33 & $0.43 \pm 0.04$ \\
\hline 5 & Gly25 - Lys28 & $0.40 \pm 0.04$ \\
\hline 6 & Gly25 - Gly29 & $0.36 \pm 0.06$ \\
\hline
\end{tabular}

Table S3 Stable intrapeptide contacts in A $\beta 25-35$ monomer bound to the pure DMPC bilayer [1]

\begin{tabular}{|c|c|c|}
\hline Rank & Contact & $\langle\boldsymbol{C}(\boldsymbol{i}, \boldsymbol{j})\rangle$ \\
\hline 1 & Gly29- Ile32 & $0.60 \pm 0.04$ \\
\hline 2 & Gly29-Gly33 & $0.50 \pm 0.05$ \\
\hline 3 & Ile31-Leu34 & $0.49 \pm 0.05$ \\
\hline 4 & Ala30-Gly33 & $0.44 \pm 0.03$ \\
\hline 5 & Ile31-Met35 & $0.43 \pm 0.06$ \\
\hline
\end{tabular}

Impact of $\mathbf{A} \boldsymbol{\beta 2 5 - 3 5}$ binding on the distribution of bilayer molecules: To understand the mechanism underlying structural perturbations in the ternary bilayer, it is important to examine the changes in the heavy atom number densities of bilayer components $n_{\alpha}(r, z)$, where $\alpha=$ DMPC, PSM, or chol. Fig. S5 shows $n_{\alpha}(r, z)$ for the three component molecules in the ternary bilayer and is discussed in the main text. 
(a)

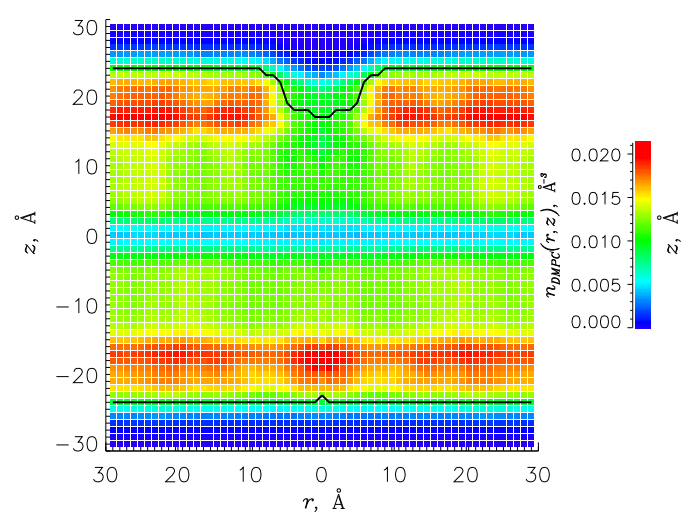

(b)

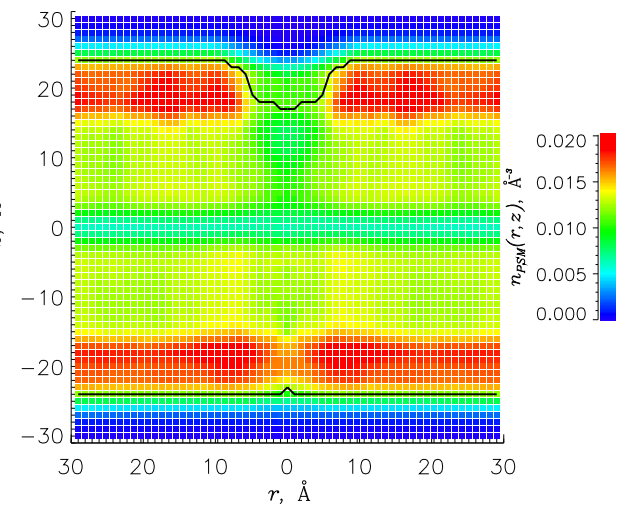

(c)

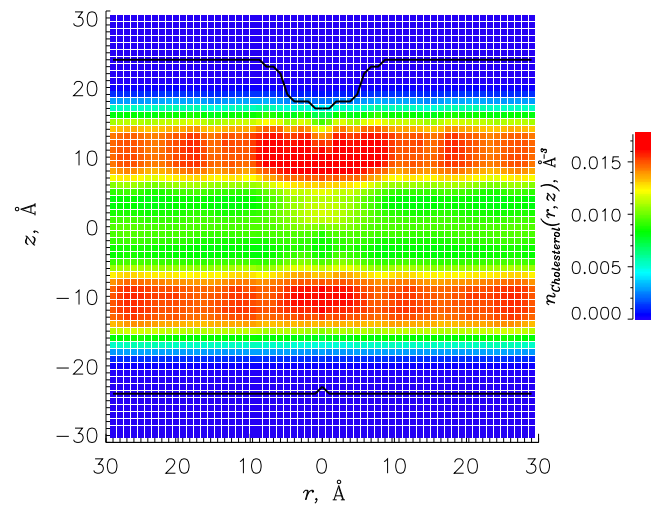

Figure S5. The heavy atom number densities of bilayer components $n_{\alpha}(r, z)$ shown as a function of the distance $r$ to the peptide center of mass and the distance $z$ to the bilayer midplane. The density cross-sections for $\alpha=$ DMPC, PSM, or cholesterol are given in panels (a-c), respectively. The bilayer boundaries $z_{b}(r)$ (from Fig. 5) are marked by solid black lines. The color scales for the number densities are shown on the right.

Impact of $\mathbf{A} \boldsymbol{\beta} 25-35$ binding on the structure of bilayer molecules: To map the changes in the structure of DMPC and PSM molecules induced by A $\beta 25-35$ binding, we compared the carbon-deuterium order parameters $\left\langle S_{C D}(i)\right\rangle$ for carbons $i$ in DMPC and PSM fatty acid tails found in the proximal and distant bilayer regions (see Methods). Fig. S6 shows that A $\beta 25-35$ peptide slightly disorders proximal lipids. The findings are analyzed in the main text. 
(a)

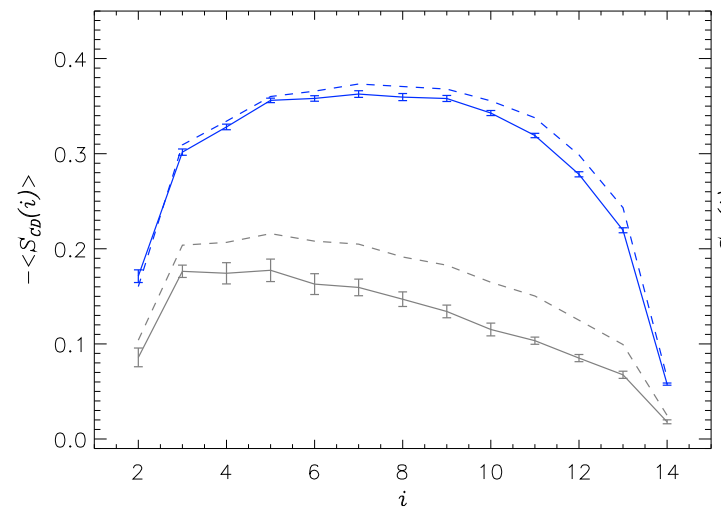

(b)

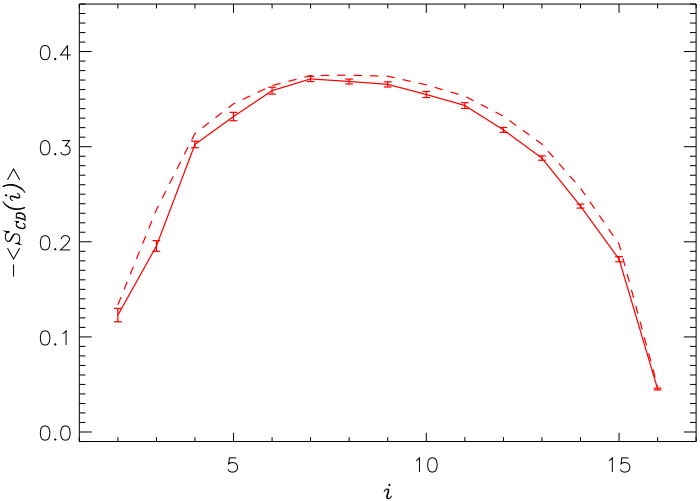

Figure S6 (a) Carbon-deuterium order parameters $\left\langle S_{C D}(i)\right\rangle$ computed for carbons $i$ in the DMPC fatty acid tails found in the proximal and distant bilayer regions (solid and dashed lines, respectively). Grey data present $\left\langle S_{C D}(i)\right\rangle$ for the DMPC bilayer [1]. (b) Similar plot as in panel (a) but computed for PSM lipids (red lines). Vertical bars represent sampling errors. Sampling errors for the distant lipids are negligible and omitted for clarity.

As an additional measure of disordering of bilayer molecules, we used their tilt angles $\gamma$ (see Methods). Fig. S7 presents the probability distributions of tilt angles $P(\gamma)$ for DMPC, PSM, and cholesterol molecules in the proximal and distant regions of the ternary bilayer. The findings are analyzed in the main text.

(a)

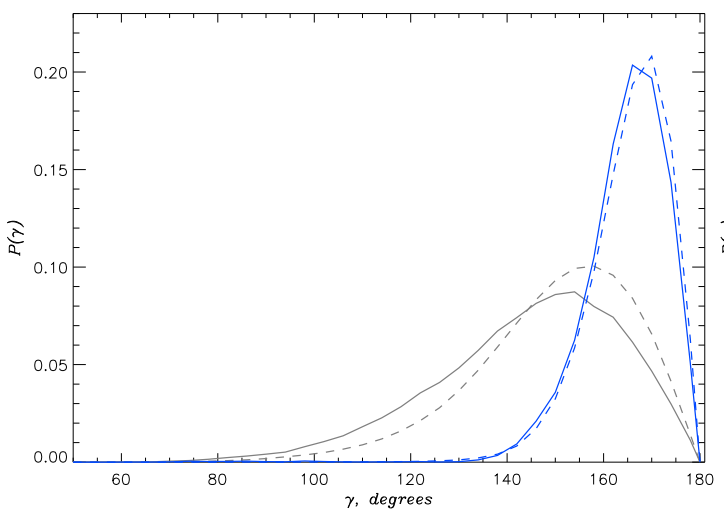

(b)

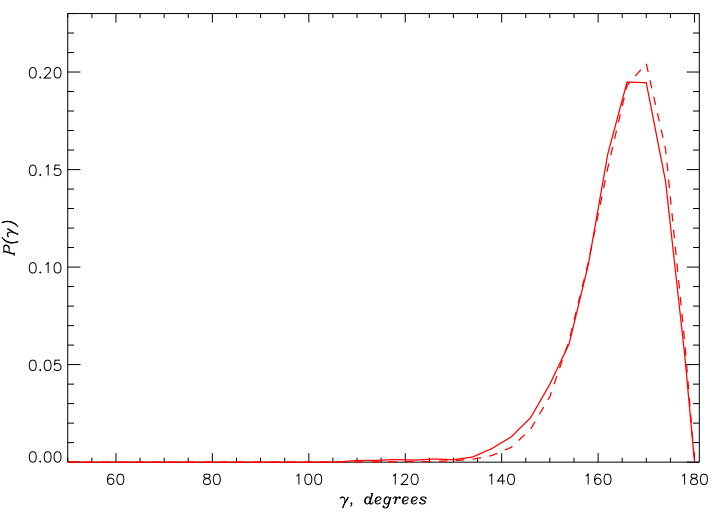

(c)

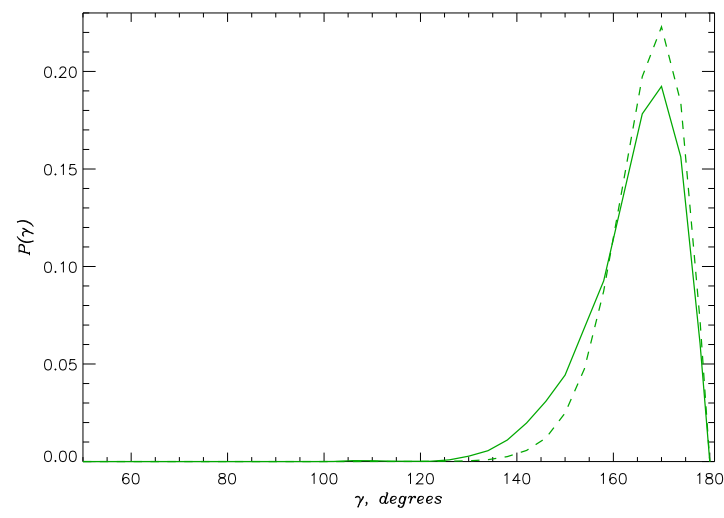


Figure S7 Probability distributions of tilt angles $P(\gamma)$ for DMPC (a), PSM (b), and cholesterol (c) molecules. Continuous and dashed lines represent proximal and distant molecules, respectively. The data in grey refer to the DMPC bilayer studied earlier [1].

Comparison of surface bound and inserted states: We have analyzed the properties of surface bound $\mathbf{S}$ and inserted I A $\beta 25-35$ states. Fig. S8 shows the helical propensity $\langle H(i)\rangle$ per residue $i$ for these two states.

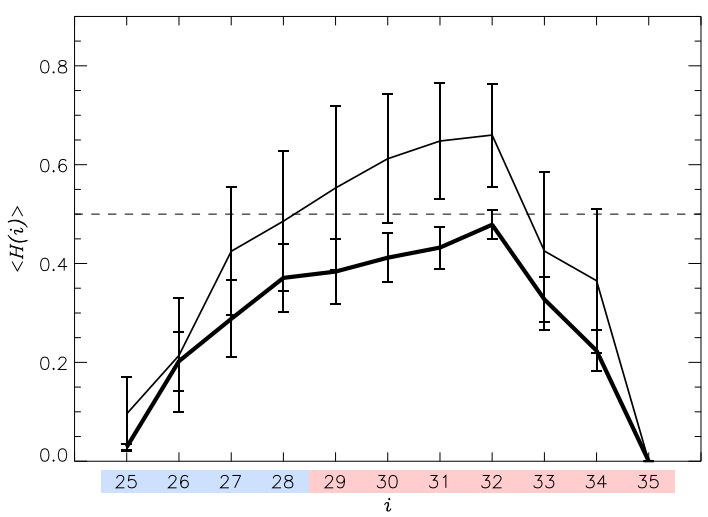

Figure S8 The fraction of helical structure $\langle H(i)\rangle$ per residue $i$ in A $\beta 25-35$ populating the surface bound $\mathbf{S}$ (thick line) and inserted I (thin line) states. Vertical bars represent sampling errors.

To compare the positions of A $\beta 25-35$ peptide within the bilayer in the states $\mathbf{S}$ and $\mathbf{I}$, we computed for these states the probabilities $P(z ; i)$ for amino acids $i$ to occur at the distance $z$ from the bilayer midplane (Fig. S9).

(a)

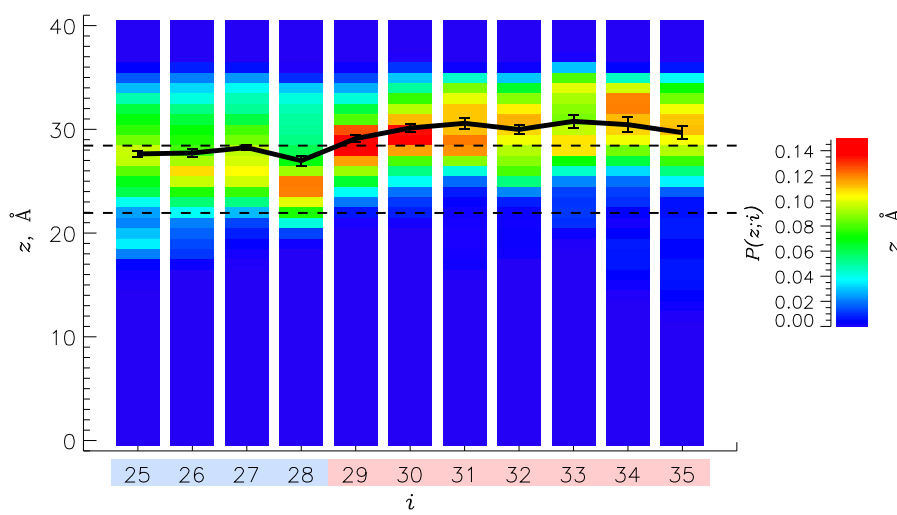

(b)

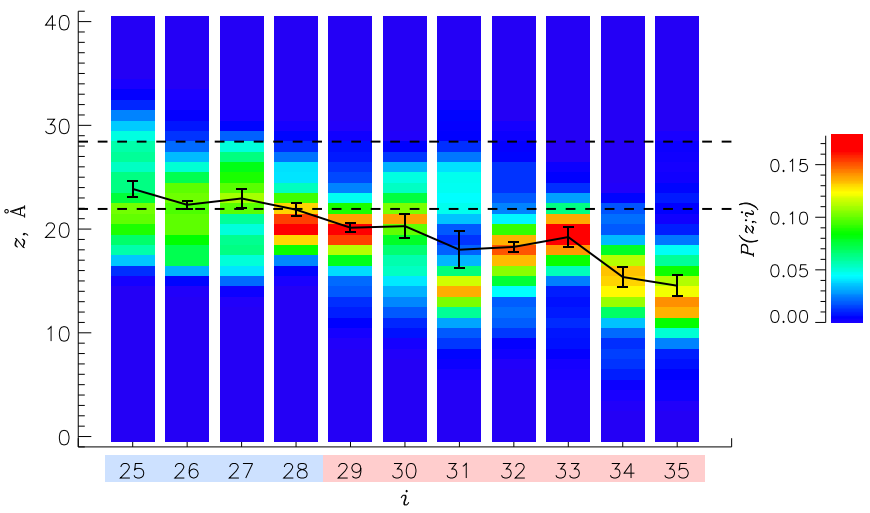

Figure S9 The probabilities $P(z ; i)$ for $\mathrm{A} \beta 25-35$ amino acids $i$ to localize at the distance $z$ from the bilayer midplane for the surface bound $\mathbf{S}$ (a) and inserted $\mathbf{I}$ (b) states. Probability color codes are shown in the right scale. Thick and thin solid lines represent the average positions of amino acids $\langle z(i)\rangle$ in $\mathbf{S}$ and $\mathbf{I}$, respectively. The dashed lines at $z_{P}$ and $z_{P}+6.5 \AA$ separate the bilayer hydrophobic core, headgroup region, and water. Vertical bars represent sampling errors.

The numbers of inserted $\left\langle N_{i}\right\rangle$, surface bound $\left\langle N_{s}\right\rangle$, and unbound $\left\langle N_{u}\right\rangle$ amino acids in the states $\mathbf{S}$ and $\mathbf{I}$ are given in Table $\mathrm{S} 4$ and analyzed in the main text. 
Table S4 Classification of amino acids in surface bound $\mathbf{S}$ and inserted $\mathbf{I}$ A $\beta 25-35$ states.

\begin{tabular}{|c|c|c|c|}
\hline State & $\left\langle N_{i}\right\rangle$ & $\left\langle N_{s}\right\rangle$ & $\left\langle N_{u}\right\rangle$ \\
\hline $\mathbf{S}$ & $0.4 \pm 0.1$ & $3.9 \pm 0.5$ & $6.7 \pm 0.7$ \\
\hline $\mathbf{I}$ & $7.6 \pm 0.2$ & $3.1 \pm 0.6$ & $0.4 \pm 0.1$ \\
\hline
\end{tabular}

The numbers of contacts between A $\beta 25-35$ amino acids $i$ and ternary bilayer molecules are shown in Fig. S10.

(a)

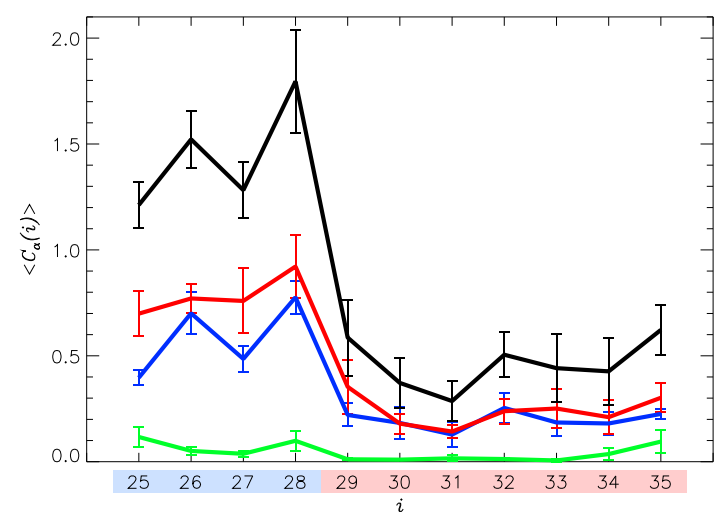

(b)

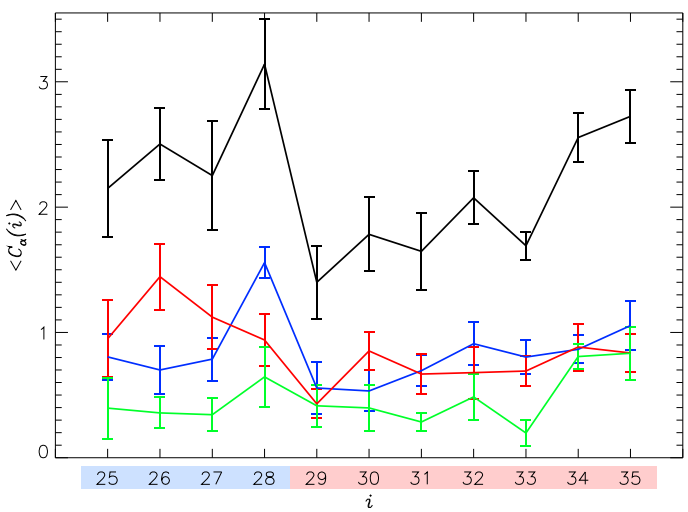

Figure S10 The numbers of contacts between A $\beta 25-35$ amino acids $i$ and all ternary bilayer structural groups $\left\langle C_{l, \alpha}(i)\right\rangle$ are shown by black line ( $\alpha=$ all). The numbers of contacts formed by amino acids with $\alpha=$ DMPC, PSM, and cholesterol groups are shown in blue, red, and green, respectively. Panels (a) and (b) present the data for the surface bound $\mathbf{S}$ and inserted $\mathbf{I}$ states. Vertical bars indicate sampling errors. R3 and R4 regions are colored following Fig. 1a.

To investigate the orientation of A $\beta 25-35$ C-terminal R4 region in the states $\mathbf{S}$ and $\mathbf{I}$, we plot in Fig. S11 the probability distributions $P\left(\gamma_{R 4}\right)$ of R4 tilt angle.

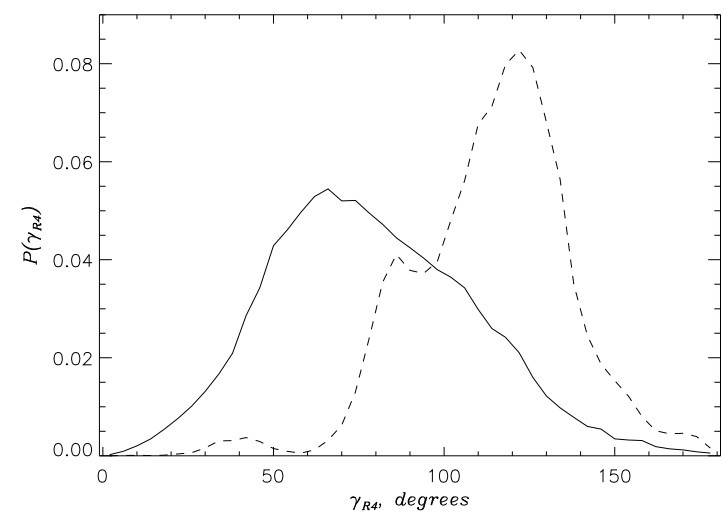

Figure S11 Probability distributions $P\left(\gamma_{R 4}\right)$ of the tilt angle $\gamma_{R 4}$ for A $\beta 25-35$ C-terminal R4 with respect to the bilayer normal computed for the $\mathbf{S}$ (solid line) and $\mathbf{I}$ (dashed line) states.

Since the surface bound $\mathbf{S}$ and inserted $\mathbf{I}$ peptides are expected to impact the ternary bilayer differently, we computed for these states the number densities of bilayer heavy atoms $n_{b l}(r, z)$ as a function of the distance $r$ from the peptide center of mass and the distance $z$ from the bilayer midplane (Fig. S12). 


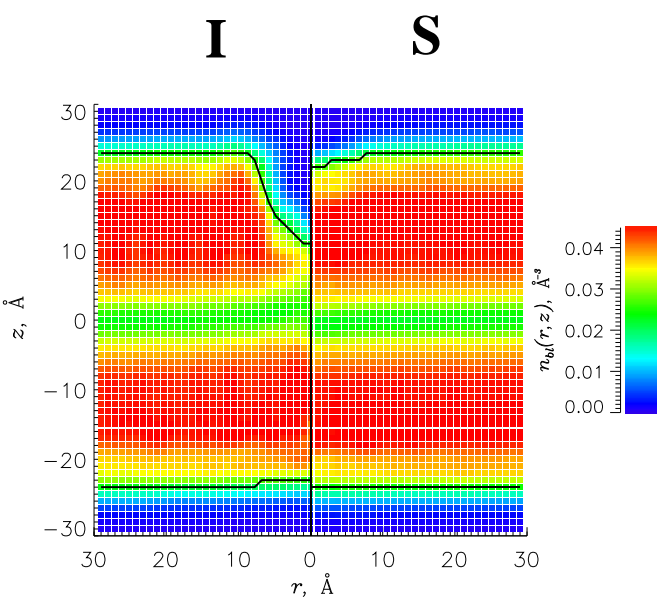

Figure S12 The number densities of bilayer heavy atoms $n_{b l}(r, z)$ shown as a function of the distance $r$ to the peptide center of mass and the distance $z$ to the bilayer midplane. The left and right halves represent $\mathbf{I}$ and $\mathbf{S}$ states. The bilayer boundaries $z_{b}(r)$ are given by solid black lines. The color scale for the number densities is shown on the right.

The implications of Figs. S8-S12 are analyzed in the main text.

Water permeation: It is important to consider the hydration of $A \beta 25-35$ monomers bound to the ternary bilayer. To this end, we have computed the number of water molecules in the first solvation shell of the peptide $N_{w}$. Fig. S13 shows the probability distribution $P\left(N_{w}\right)$ for A $\beta 25-35$ peptides binding to the ternary and DMPC bilayers. Two peaks in $P\left(N_{w}\right)$ reflect the coexistence of surface bound $\mathbf{S}$ and inserted $\mathbf{I}$ states.

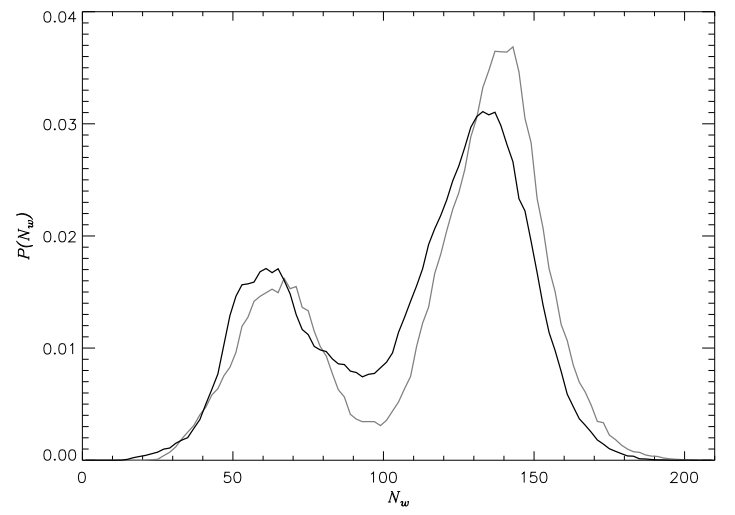

Figure S13 Probability distributions $P\left(N_{w}\right)$ of the numbers of water molecules $N_{w}$ in the first solvation shell of A $\beta 25-35$ peptide bound to the ternary and DMPC bilayers. The respective data are in black and grey.

[1] Smith, A. K. and Klimov, D. K. (2018) Binding of cytotoxic A $\beta 25-35$ peptide to the DMPC lipid bilayer. J. Chem. Inform. Model. 58, 1053-1065.

[2] Han, M. and Hansmann, U. H. E. (2011) Replica exchange molecular dynamics of the thermodynamics of fibril growth of Alzheimer's A 342 peptide. J. Chem. Phys. 135, 065101. 